\title{
Design and Validation of Differential Braking Controllers for Sport Utility Vehicles Considering the Interactions of Driver and Control System
}

\section{Shenjin Zhu and Yuping He*}

Department of Automotive, Mechanical and Manufacturing Engineering, University of Ontario Institute of Technology, Canada

*Corresponding author: Yuping He, Department of Automotive, Mechanical and Manufacturing Engineering, University of Ontario Institute of Technology, Oshawa, Ontario, Canada, Email: yuping.he@uoit.ca

\section{Research Article \\ Volume 2 Issue 3}

Received Date: May 26, 2018

Published Date: June 14, 2018

DOI: $10.23880 /$ eoij-16000155

\section{Abstract}

This paper introduces the design and validation of a differential braking controller for sport utility vehicles (SUVs) with driver-in-the-loop real-time simulations. SUVs are designed with high ground clearance, which is a main reason for their high rollover rate. A nonlinear 3 degrees-of-freedom (DOF) SUV model is generated to design a differential braking controller. The desired states are determined using a 2-DOF bicycle model and the lane-keeping control results derived from vehicle velocity and road curvature. The actual vehicle states of the 3-DOF model may deviate from the desired ones. A sliding model controller (SMC) is designed to minimize the state error to improve the performance measures, e.g., yaw stability. The SMC controller designed in LabVIEW is integrated with a virtual SUV generated in CarSim for cosimulations. The controller is first examined in the emulated sine-with-dwell maneuver specified in FMVSS 126. The SUV performance depends not only on the control strategy, but also on its interaction with the human driver. To study the interaction of the driver and the controller, the overall system is simulated using driver-software-in-the-loop (DSIL) realtime simulations under a double-line-change (DLC) maneuver. The simulations show that, even equipped with the electronic stability control (ESC) system, the driver still plays an important role in the vehicle dynamics. The simulations demonstrate the effectiveness of the proposed differential braking controller, and the research discloses important interactions of driver and ESC system.

Keywords: Design and Validation; Differential Braking Control; Interactions of Driver and Control System; CoSimulations; Sport Utility Vehicles 


\section{Ergonomics International Journal}

\section{Introduction}

Lateral stability is an important part of road vehicles with high center of gravity (CG), e.g., sport utility vehicles (SUVs) and trucks [1,2]. It is shown that rollover accidents account for about $80 \%$ of non-collision fatal crashes, among which $70 \%$ are associated with light trucks, including SUVs and pickups [3]. SUVs have been increasing since 1990s. High CG of SUVs may be the main reason for the higher rollover accident rate. Studies indicate that active safety systems (ASSs), e.g., electronic stability control (ESC) [4], are able to improve the lateral stability of vehicles and reduce highway accident rates [5]. The past two decades has witnessed the advancement of vehicle ASSs that prevent vehicles from dangerous accidents [6].

Lateral stability control has attracted the attention of researchers [7,8]. SUV stability control by means of applying corrective yaw moment can reduce the deviation from the desired vehicle behaviors. The control of yaw moment can be achieved using a variety of approaches, e.g., torque distribution control, and differential braking control (DBC). To date, DBC has been applied to road vehicles due to their cost-effectiveness $[9,10]$. With the DBC technique, the required yaw moment can be achieved by manipulating the braking effects at different wheels, differentiating braking pressures in the left/right and/or front/rear wheel cylinders [11]. To validate and improve DBC systems, field and road tests of real physical prototypes are not dispensable [12]. However, at the initial development stage, the field and road tests can be difficult, time-consuming, dangerous, and costly. Therefore, real-time simulations have been applied to assess control performance prior to in-vehicle field and road tests [13]. These real-time simulations are often based on conventional open-loop approaches, investigating the control performance under given driving inputs without considering the effects of drivers, which may play a destabilizing part in the vehicle-human-road system. The overall performance of a road vehicle depends not only on the controller, but also on its interaction of human driver and road. Thus, open-loop real-time simulation approaches may not adequately address the driver-controller interactions and the overall performance of a vehicle with ASS.

In this paper, we design and validate a DBC controller for SUVs using the driver-software-in-the-loop (DSIL) real-time simulations. Firstly, we generate a nonlinear yaw-plane vehicle model with 3 DOF, and design the DBC controller using the nonlinear model. Secondly, to validate and improve the controller design, the real-time SUV model is reconstructed and the controller is reformulated in the CarSim and LabVIEW packages, respectively. With integration of the controller and the CarSim SUV model through the interface between the two software packages, the DSIL real-time simulation is implemented on the DSIL platform in the Multidisciplinary Vehicle Systems Design Laboratory at the University of Ontario Institute of Technology (UOIT). The rest of the paper is organized as follows. The models of SUV lateral dynamics are introduced in Section 4.1. Design optimization of the DBC controller is presented in Section 5. In Section 6, the configuration of the DSIL platform and integration of the real-time SUV model and the controller are described in detail. In Section 7, simulation results are analyzed and discussed. Finally, conclusions are drawn in Section 8.

\section{Vehicle Models}

Three vehicle models are used in this research: 1) a 3DOF nonlinear yaw-plane model, 2) a 2-DOF linear bicycle model, and 3) a 14-DOF nonlinear CarSim model. The 3DOF nonlinear model is applied to compute the control command. The bicycle model is used for generating the desired yaw-rate and sideslip angle trajectories. The CarSim model serves as a real-time virtue vehicle to validate the proposed controller and investigate the interactions of driver-vehicle-controller-road.

\section{3-DOF Nonlinear Yaw-Plane Model}

Figure 1 shows the 3-DOF yaw-plane model, where, F, T P, $\alpha$, and $\rho$ denote the tire force, torque, wheel cylinder brake pressure, wheel sideslip angle, and the brake pressure proportional ratio between the front and rear wheel, respectively, with subscript b for braking, $d$ for driving, $\mathrm{f}$ for front, $\mathrm{r}$ for rear/right, $\mathrm{l}$ for left, $\mathrm{x}$ for longitudinal, and y for lateral, and $u, v, \Upsilon, \beta$ denoting the longitudinal velocity, lateral velocity, yaw rate, and sideslip angle of the vehicle at the center of gravity (CG), $\mathrm{V}, \mathrm{V}_{\mathrm{f}}, \mathrm{V}_{\mathrm{r}}$ representing the velocities at the CG, front and the rear axles, and a, b, d meaning the distances from the CG to the front and rear axles and the wheel track of the vehicle, and $\delta$ the steering angle. It is assumed that: the self-alignment torque of wheels is negligible; the steering angle and sideslip angle for the bicycle model are small; the payload of the SUV is symmetrically distributed along longitudinal and lateral directions of the vehicle body; this SUV uses front wheel drive; and the dynamics of the wheels is negligible. 


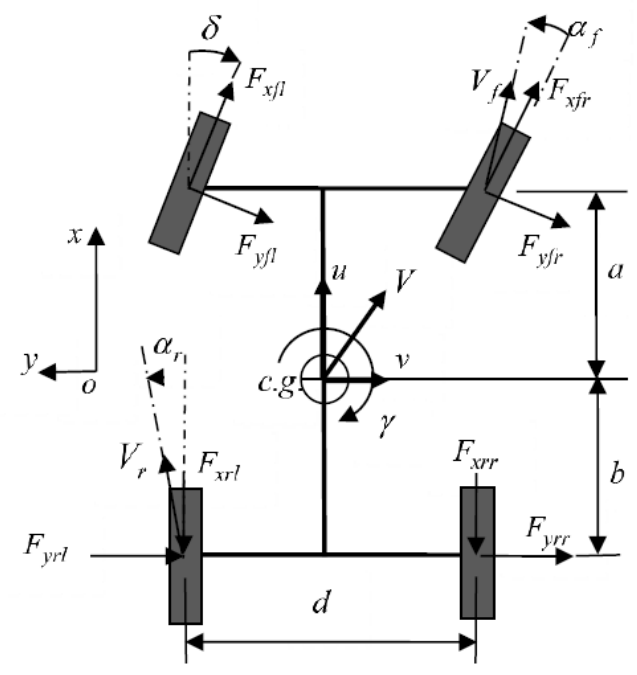

Figure 1: The 3-DOF yaw-plane model of the sport utility vehicle.

The equations of motion can be obtained by using the Newton's second law as

$$
\begin{gathered}
x=f(x)+b M_{\mathrm{Y} b} \\
y=I[\beta \mathrm{Y}]^{T}
\end{gathered}
$$

Where

$$
\begin{gathered}
X=\left[\begin{array}{cc}
\beta & \gamma
\end{array}\right]^{T}, \mathrm{~b}=\left[\begin{array}{ll}
0 & 1
\end{array}\right]^{T}, f=\left[\begin{array}{ll}
f_{1} & f_{2}
\end{array}\right]^{T} \\
f_{1}=-\left(\frac{v^{2}}{u^{2}}+1\right) \gamma+\frac{1}{m u}\left(\sin \delta-\frac{v}{u} \cos \delta\right) F_{x f}+\frac{v}{m u^{2}} F_{x r}+\frac{1}{m u}\left(\cos \delta-\frac{v}{u} \sin \delta\right) F_{y f}-\frac{1}{m u} F_{y r}, \\
f_{2}=\frac{a}{I_{z}} F_{x f} \sin \delta+\frac{a}{I_{z}} F_{y f} \cos \delta-\frac{b}{I_{z}} F_{y r}, F_{x f}=F_{x f l}+F_{x f r}, F_{x r}=F_{x r l}+F_{x r r}, \\
F_{y f}=F_{y f l}+F_{y f r}, F_{y r}=F_{y r l}+F_{y r r} \\
M_{\gamma b} \equiv \frac{d}{2 I_{z}}\left(F_{x f r}-F_{x f l}\right) \cos \delta-\frac{d}{2 I_{z}}\left(F_{x r r}-F_{x r l}\right),
\end{gathered}
$$

and I denotes the identity matrix, $M_{\gamma b}$ the external yaw moment, $\mathrm{m}$ the total mass of the vehicle, and $\mathrm{I}_{\mathrm{z}}$ the vehicle moment of inertia about yaw axis. The longitudinal and lateral forces of wheels are determined using the Dugoff's tire model [14] in terms of the wheel normal force $F_{z}$ and wheel cornering and slip coefficients $\mathrm{c}_{\alpha}$ and $\mathrm{c}_{\sigma}$.
The yaw dynamics can be manipulated using direct yaw moment control (DYC), in which a yaw moment is introduced using differential braking forces on the left and right wheels. The braking forces can be determined by analyzing the longitudinal forces of wheels as

$$
\mathrm{T}_{b f l}=\frac{1}{2} T_{d}-r_{w} F_{x f l}, T_{b f r}=\frac{1}{2} T_{d}-r_{w} \mathrm{~F}_{x f r}, T_{b r l}=r_{w} F_{x r l}, T_{b r r}=r_{w} F_{x r r}
$$


where

$$
\begin{aligned}
& T_{b f l}=K_{b f} P_{b f l} \\
& T_{b f r}=K_{b f} P_{b f r} \\
& T_{b r l}=K_{b r} P_{b r l} \\
& T_{b r r}=K_{b r} P_{b r r}
\end{aligned}
$$

$\mathrm{K}_{\mathrm{bf}}$ and $\mathrm{K}_{\mathrm{br}}$ are the brake gains of the front and rear wheels, respectively, $r_{w}$ is the effective radius of the wheel, and the brake pressures of the rear wheels $\mathrm{P}_{\mathrm{brl}}$ and $\mathrm{P}_{\mathrm{brr}}$ are determined from the brake pressures of the front wheels $\mathrm{P}_{\mathrm{bfl}}$ and $\mathrm{P}_{\mathrm{bfr}}$, using the proportioning technique with threshold pressure $p_{0}$. The external yaw moment is manipulated from the definition in Equation (1) in terms of the brake pressures on the front wheels as

$$
\mathrm{M}_{\gamma b}=\left[d /\left(2 r_{w} I_{z}\right)\right]\left[\left(K_{b r \rho l}+K_{b f} \cos \delta\right) P_{b f l}-\left(K_{b r \rho r}+K_{b f} \cos \delta\right) P_{b f r}\right]
$$

\section{2-DOF Linear Bicycle Model}

The relationship of the steering angle, tire sideslip angle and the yaw rate can be clearly represented using the 2-DOF bicycle model [14] as shown in Figure 2. It is assumed that the radius of the road curvature (R) is much larger than the wheelbase $(\mathrm{L}=\mathrm{a}+\mathrm{b})$, and a linear geometrical relationship can be constructed among the steering angle and the tire sideslip angles $\delta=\mathrm{L} / \mathrm{R}-\left(\alpha_{\mathrm{f}}-\alpha_{\mathrm{r}}\right)$. In a steady-state steering maneuver, the vehicle is in a pseudo-equilibrium state, and the total lateral forces form a centripetal force for a circular motion. The lateral tire force is a liner function of the cornering stiffness $\mathrm{C}_{\alpha}$. The steady-state steering angle and sideslip angel are expressed in terms of the velocity of the vehicle and radius of the curvature as [14] as

$$
\delta_{s s}=L / R-K_{u} V^{2} / R, \beta_{s s}=b / r-\left(m_{r} V^{2}\right) /\left(2 C_{\alpha r} R\right)
$$

where $K_{u}=m_{f} /\left(2 C_{\alpha f}\right)-m_{r} /\left(2 C_{\alpha r}\right)$, denotes the under steer gradient with $m_{f}=(b / \mathrm{L}) \mathrm{m}$ and $\mathrm{m}_{r}=(a / L) m$. The desire yaw rate generated by the pair $(\mathrm{V}, \mathrm{R})$ can be expressed in terms of the steady-state steering angle as

$$
\gamma_{\text {des }}=\left(V \delta_{s s}\right) /\left(L-K_{u} V^{2}\right), \beta_{\text {des }}=\left[b-m_{r} V^{2} /\left(2 C_{a r}\right)\right] \delta_{s s} /\left(\mathrm{L}-\mathrm{K}_{u} V^{2}\right)
$$

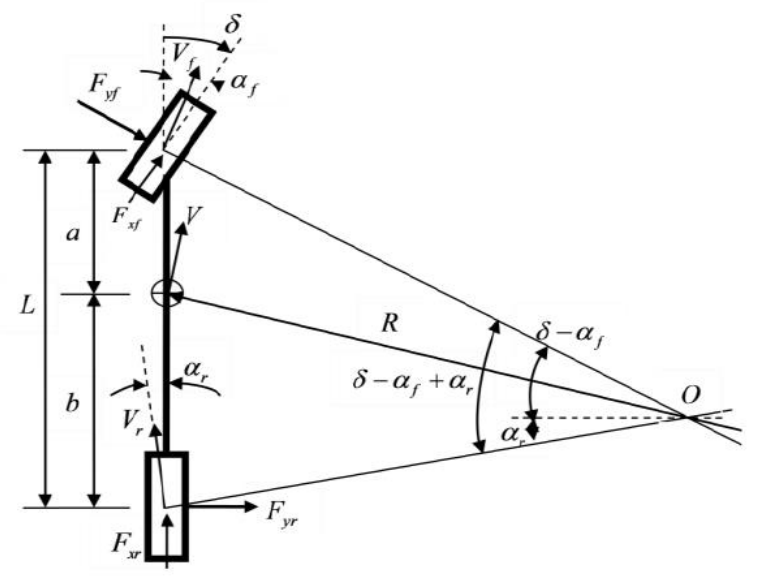

Figure 2: The 2-DOF bicycle model.

\section{4- DOF Nonlinear CarSim Model}

CarSim is a software package by Mechanical Simulation Corporation, and the package is widely used for modelling and simulating the dynamic behavior of passenger cars
[15]. In CarSim, the SUV is modeled as an interconnected rigid multi-body system consisting of a sprung mass, two axles, and four rotating wheels. The power train system and steering system have been lumped into the sprung mass considering the respective kinematic relationships. 


\section{Ergonomics International Journal}

The CarSim model is developed with 14-DOF, including 6DOF for the sprung mass, 2-DOF for each axle, and one rotation DOF for each wheel. The motions of the rigid bodies are governed with ordinary differential equations, which take inputs, e.g., driving torque from the power train, braking torque from the braking system, roll moment from the suspension systems, and frictional forces from the tire/road interfaces and aerodynamic forces. All these inputs have nonlinear properties in nature. They may be modeled using lookup tables containing experimental data collected from academia and the automotive industry.

\section{SMC Controller Design}

The controller design is formulated as a tracking problem as shown in Figure 3. The output of the plant (i.e., the SUV) $[\beta \gamma]^{T}$ is compared with the target $\left[\beta_{\text {des }} \gamma_{\text {des }}\right]^{T}$. The error is fed into a controller to generate a yaw moment; it is then converted into brake pressures of the front and rear wheels. The controller and the converter form a compensator for the yaw dynamics of the SUV. Due to the nonlinear nature of the SUV and wide application of approximations, a robust nonlinear controller, sliding mode controller (SMC) [16], is applied.

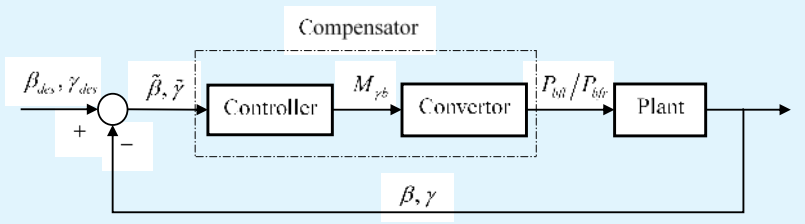

Figure 3: The block diagram of yaw dynamics control system.

With the nonlinear yaw-plane SUV model described in Equation (1), a sliding mode surface in terms of the tracking error is defined as

$$
s=\left(\gamma_{\text {des }}-\gamma\right)+\phi\left(\beta_{\text {des }}-\beta\right)=0
$$

where $\phi$ is a positive weighting factor. Based on the stability condition, $\dot{s}=-\eta s(\eta>0)$, a control law can be obtained

$$
M_{\gamma b}=\phi f_{1}-f_{2}+\eta\left(\gamma_{d e s}-\gamma\right)+\eta \phi\left(\beta_{d e s}-\beta\right)
$$

The control law can be converted to braking pressures using Equation (3), a linear combination of moments created by the left and right wheels $M_{\gamma b}=M_{l}+M_{r}$. Since the brake pressures are non- negative, and the steering angle takes a value in the range $(-\pi / 2, \pi / 2)$, with the current coordinate sign convention, the yaw moments induced by differential braking satisfy the constraints: yaw moment of left wheels $\mathrm{M}_{1} \geq 0$ and yaw moment of right wheels $M_{r} \leq 0$. Thus, the application of DBC is scheduled as applying brakes on left wheels when $\mathrm{M}_{\gamma \mathrm{b}}>0$ and on right wheels when $M_{\gamma b}<0$. The application of brake proportioning technique makes the control of yaw moment simply through manipulating the brake pressures only on front wheels. The controlled system is shown diagrammatically in Figure 4.

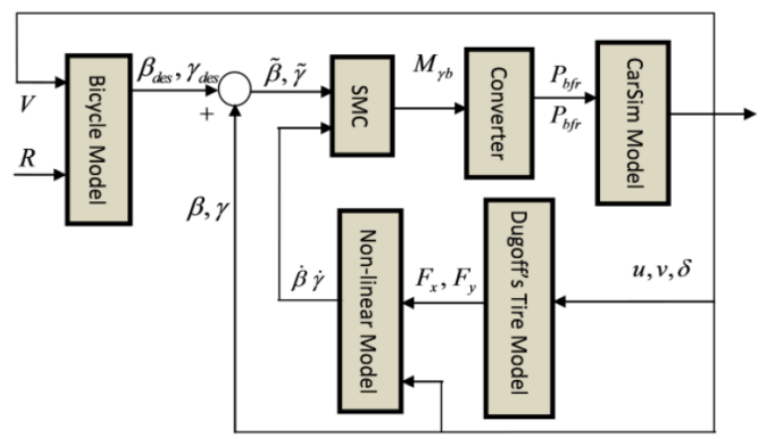

Figure 4: The block diagram for the SMC controlled SUV.

\section{Real-Time Simulations Using UOIT DSIL Platform}

The controller introduced in the previous section is examined on the UOIT DSIL platform. The DSIL platform provides an easy-to-use virtual testing environment, which integrates the driver, controller, virtual vehicle, road and the visual display in a real-time working mode. The configuration of the DSIL platform is illustrated in Figure 5.

The DSIL platform consists of a host computer, a target computer, an animator computer, three monitors, a realtime operation system by National Instruments, communication links, and $\mathrm{I} / \mathrm{O}$ boards. The platform permits the interactions between the driver and controller in such a way that a virtual vehicle is driven by a driver under a specified test maneuver. 


\section{Ergonomics International Journal}

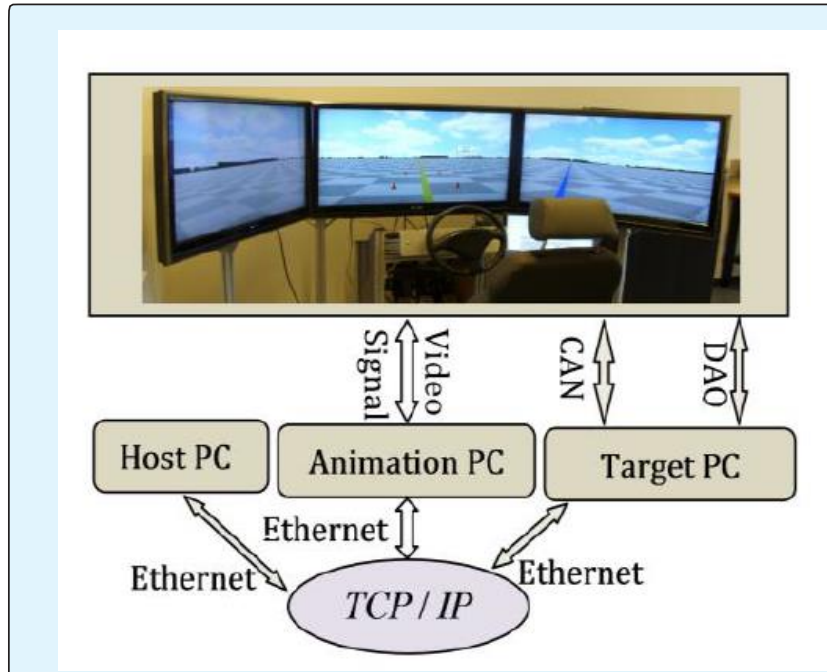

Figure 5: The configuration of the UOIT DSIL platform.

One of the key issues in setting up the virtual test environment is to develop the real-time version of the controller in LabVIEW, and real-time SUV model in CarSim. The integration of the controller and vehicle model involving LabVIEW and CarSim packages is implemented.

In the DBC system, all the measured vehicle states are obtained from the real-time CarSim model. The SMC controller and converter are reconstructed in LabVIEW. With the integration and synchronization of the vehicle model and controller in real-time on the DSIL platform, the interactions among the human driver, controller, virtual vehicle and the road can be fully investigated for the design and validation of the DBC system.

\section{Simulation Results and Discussion}

To simulate the SUV dynamics, the vehicle system parameters take the values listed in Table 1 . The DBC controller designed in Section 2 is evaluated by simulating the following two types of test:

(1) An open-loop test, the sine-with-dwell test specified by the US Federal Motor Vehicle Safety Standard (FNVSS), No. 126 (hereafter called FMVSS 126).

(2) A closed-loop test, the driver-software-in-the-loop real-time simulations conducted on the UOIT DSIL platform.

The sine-with-dwell test is mainly used to investigate the performance of the DBC controller alone, while the DSIL real-time simulations are conducted to examine the overall performance of the driver-controller-road integration.

\begin{tabular}{|c|c|c|c|}
\hline Par. & Value & Par. & Value \\
\hline $\mathrm{C}_{\alpha}$ & $12 \mathrm{rad}^{-1}$ & $\mathrm{C}_{\sigma}$ & 19 \\
\hline $\mathrm{I}_{\mathrm{z}}$ & $2059.2 \mathrm{~kg} . \mathrm{m}^{2}$ & $\xi$ & 0.3 \\
\hline $\mathrm{K}_{\mathrm{bf}}$ & $300 \mathrm{~N} / \mathrm{MPa}$ & $\mathrm{K}_{\mathrm{br}}$ & $150 \mathrm{~N} / \mathrm{Mpa}$ \\
\hline $\mathrm{a}$ & $1.05 \mathrm{~m}$ & $\mathrm{~b}$ & $1.55 \mathrm{~m}$ \\
\hline $\mathrm{d}$ & $1.565 \mathrm{~m}$ & $\mathrm{~m}$ & $1610 \mathrm{~kg}$ \\
\hline $\mathrm{P}_{\mathrm{o}}$ & $2.0 \mathrm{MPa}$ & $\mathrm{r}_{\mathrm{w}}$ & $0.38 \mathrm{~m}$ \\
\hline
\end{tabular}

Table 1: Vehicle and controller parameters.

\section{Simulated Sine-with-Dwell Tests}

The sine-with-dwell procedure is an indicative test maneuver for the compliance of FMVSS 126 [17]. It involves two tests, i.e., slowly increasing steer (SIS) maneuver and sine-with-dwell test. The SIS maneuver is first performed to determine the fundamental amplitude (A) for the sine-with-dwell tests. Then, the sine-withdwell test is carried out and the responses, e.g., the steering wheel angle (SWA), lateral acceleration, vehicle speed, and the yaw rate, are recorded and analyzed. The test is terminated when the stopping criteria are reached.

SIS maneuver: The SIS maneuver is a preliminary test for determining the fundamental amplitude (A) of sine-withdwell SWA signals. In this maneuver, a linearly increasing SWA from zero to $30 \mathrm{deg}$ at the rate of $13.5 \mathrm{deg} / \mathrm{s}$ is applied at a constant vehicle forward speed of $80 \pm 2 \mathrm{~km} / \mathrm{h}$. The predetermined SWA is requested to achieve a lateral acceleration of $0.3 \mathrm{~g}$, with acknowledgement of a linear relationship between the SWA and lateral acceleration from to $0.1 \mathrm{~g}$ to $0.375 \mathrm{~g}$. The SWA at $0.3 \mathrm{~g}$ is considered as the basic amplitude (A).

Sine-with-Dwell maneuver: Two series of sine-withdwells are performed, i.e., clockwise first half cycles and counter-clockwise first half cycles. The test starts with counter-clockwise direction with an initial amplitude 1.5 A. In each test, when coasting at $80 \pm 2 \mathrm{~km} / \mathrm{h}$, the SUV is excited by a $0.7 \mathrm{~Hz}$ Sinewave SWA with 0.5 seconds delay from the second wave-peak. Several tests are carried out with an amplitude increment of $0.5 \mathrm{~A}$ from the previous one. The number of tests required before the second series depends on the vehicle dynamic responses evaluated with the passing and stopping criteria.

Passing and stopping criteria: The main concerns are the SUV yaw dynamics and responsiveness identified respectively by the yaw rate and lateral displacement. The passing and stopping criteria are thus defined as the yaw 


\section{Ergonomics International Journal}

rate and lateral displacement at specified times with discrimination on the vehicle gross weight (VGW) and SWA amplitude $\left(\delta_{\mathrm{A}}\right)$. The passing criteria are: 1$)$ yaw rate satisfying $\gamma(2.929) \leq 35 \% \gamma_{\text {peak }}$ and $\gamma(3.679) \leq 20 \% \gamma_{\text {peak, }}$ 2) lateral displacement complying y(1.07) $\geq 1.52 \mathrm{~m}$ for $\delta_{\mathrm{A}} \geq 5 \mathrm{~A}$ and VGM $\leq 350 \mathrm{~kg}$; and 3) lateral displacement meeting $y(1.07) \geq 1.52 \mathrm{~m}$ for $\delta_{A} \geq 5 \mathrm{~A}$ VGM $>3500 \mathrm{~kg}$. The stopping criteria are defined according to the dynamic response and the SWA amplitude. $\delta_{\mathrm{A}}$ as: Test fails the passing criteria, if the amplitude reaches $\delta_{\mathrm{A}}>270 \mathrm{deg}$ or $\delta_{\mathrm{A}}>6 \mathrm{~A}$. The sine-with-dwell test is performed following the procedure offered in CarSim [18]. The test results illustrate that the controlled vehicle has successfully passed the test while the baseline vehicle failed the test on the 5th run in terms of the yaw criteria as seen in Figure 6.

\section{DSIL Real-Time Simulations}

Two test procedures, i.e., double-lane-change (DLC) and accident avoidance (AA), are simulated on the UOIT DSIL for real-time simulations with two drivers. One of the two drivers is an inexperienced driver with less than 10 hours driving experience on the DSIL platform, and the other is an experienced driver with more 100 hours driving experience. In the DLC tests, the driver-dependent performance and the robustness of the DBC controlled system are examined. In the AA tests, drivers' overreaction in an emergency situation is investigated.

DLC Simulations for driver-dependent performance: The purpose of the DSIL real-time simulation is to examine the combined performance of the drivercontroller integration, specifically, how the performance depends on the driver's actions. The DLC test procedure is specified as follows. Over the test, the vehicle forward speed maintains constant at $100 \mathrm{~km} / \mathrm{h}$. The test track is $80 \mathrm{~m}$ long with a $3.5 \mathrm{~m}$ lateral offset. The surface of the test track has a friction coefficient of $\mu_{0}=0.85$. For every simulated test, each driver drives the virtual SUV with the DBC controller 20 times. Each simulated test takes about 10 seconds. The simulation results are investigated in terms of vehicle yaw rate and side slip angle.

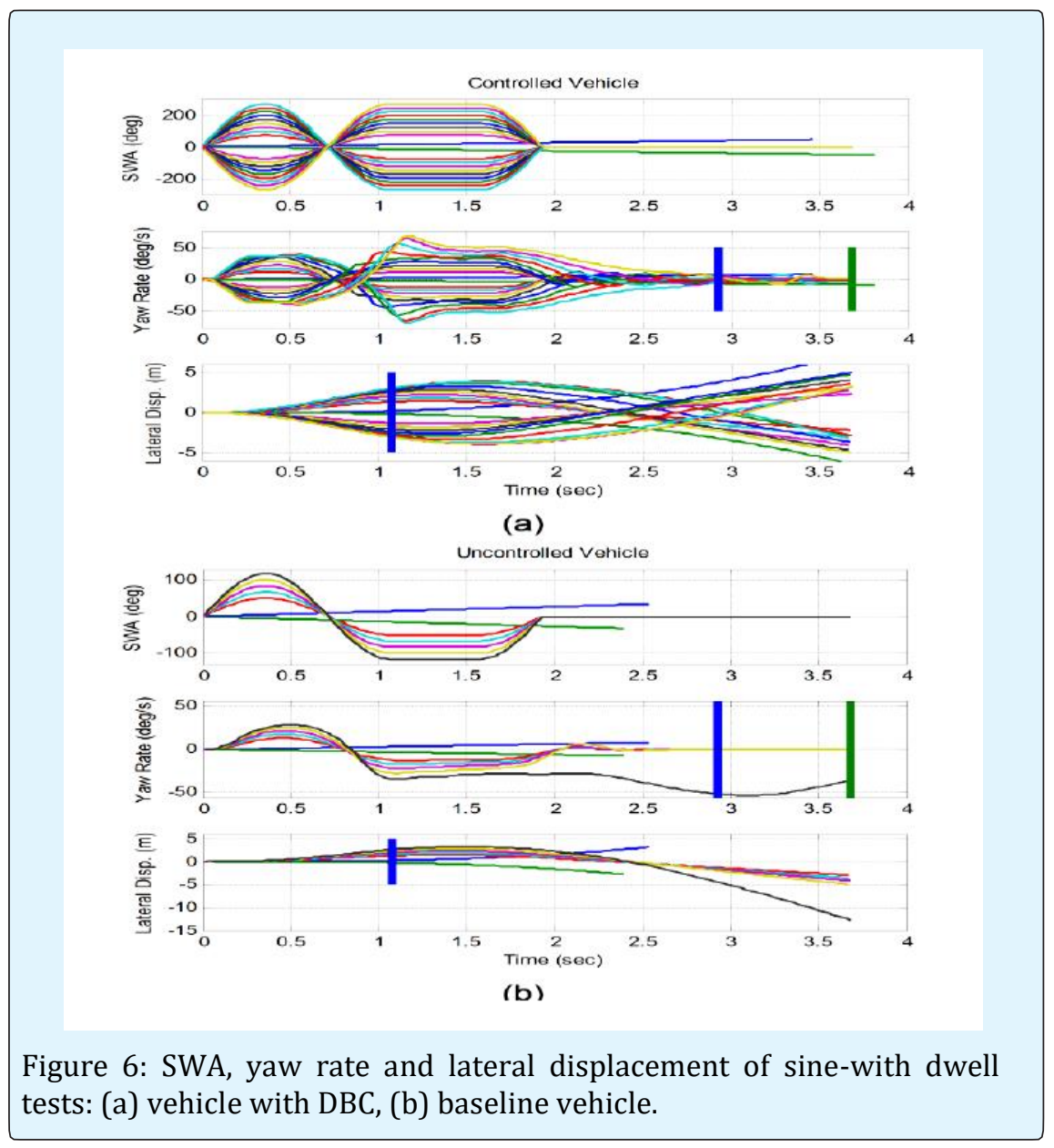

Shenjin Zhu and Yuping He. Design and Validation of Differential Braking Controllers for Sport Utility Vehicles Considering the Interactions of Driver and Control System. Copyright $@$ Shenjin Zhu and Yuping He. Ergonomics Int J 2018, 2(3): 000155. 
Figure 7 illustrates the simulation results of the yaw rate, target yaw rate, and tracking errors derived from 20 DLC tests of the SUV with the DBC controller driven by the inexperienced driver. It should be noted that: in Figure 7, all of the results for the 20 test runs are shown; the 20 test runs are divided into 4 groups, and each group involves five test runs; for each group of five test runs, the respective result is denoted by a curve in a specified color. The peak yaw rate of the first five runs reaches $50 \mathrm{deg} / \mathrm{s}$. With building-up driving experience, the peak yaw rate drops to approximate $25 \mathrm{deg} / \mathrm{s}$ for the second five runs, and continuously drops to $20 \mathrm{deg} / \mathrm{s}$ for the last five runs.
A similar trend is also observed in the tracking error measurements. The peak tracking error decreases from $15 \mathrm{deg} / \mathrm{s}$ for first few tests to $1.5 \mathrm{deg} / \mathrm{s}$ for the last few runs. It is indicated that the performance of the SUV with the DBD controller is improving with the building-up driving experience. The driver's action directly influences the performance of the controlled SUV through generating target yaw trajectories. An inexperienced driver, when facing an emergency situation, is more likely to generate a target trajectory which is harder for a controller to track and a larger tracking error can be expected.

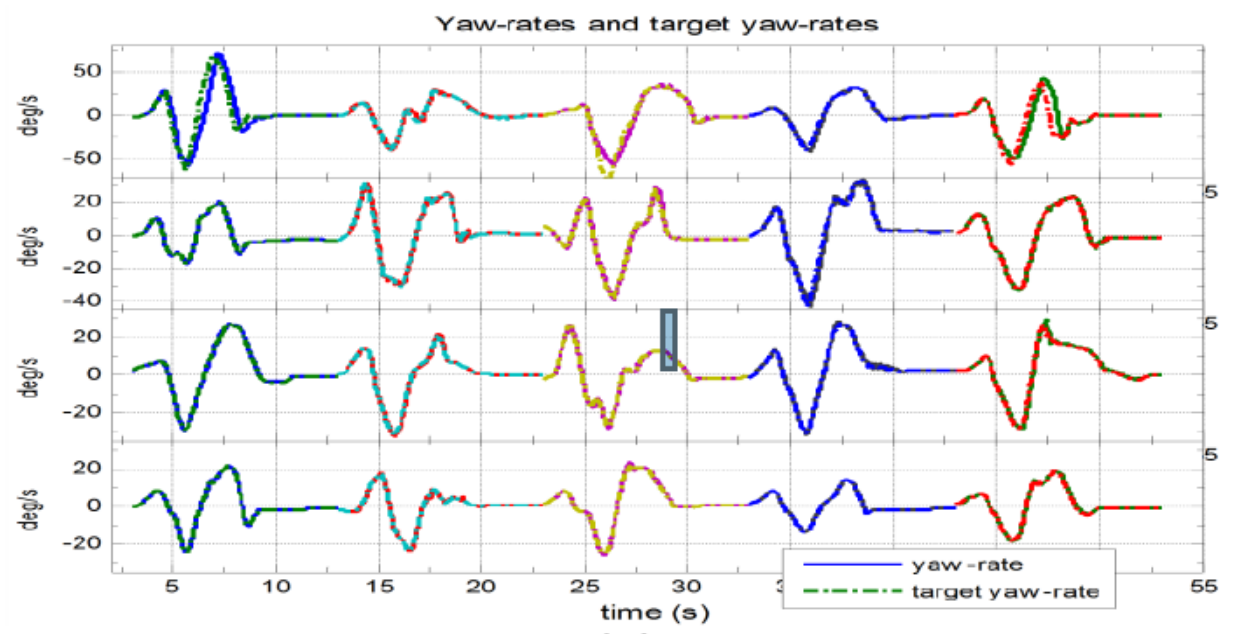

(a)

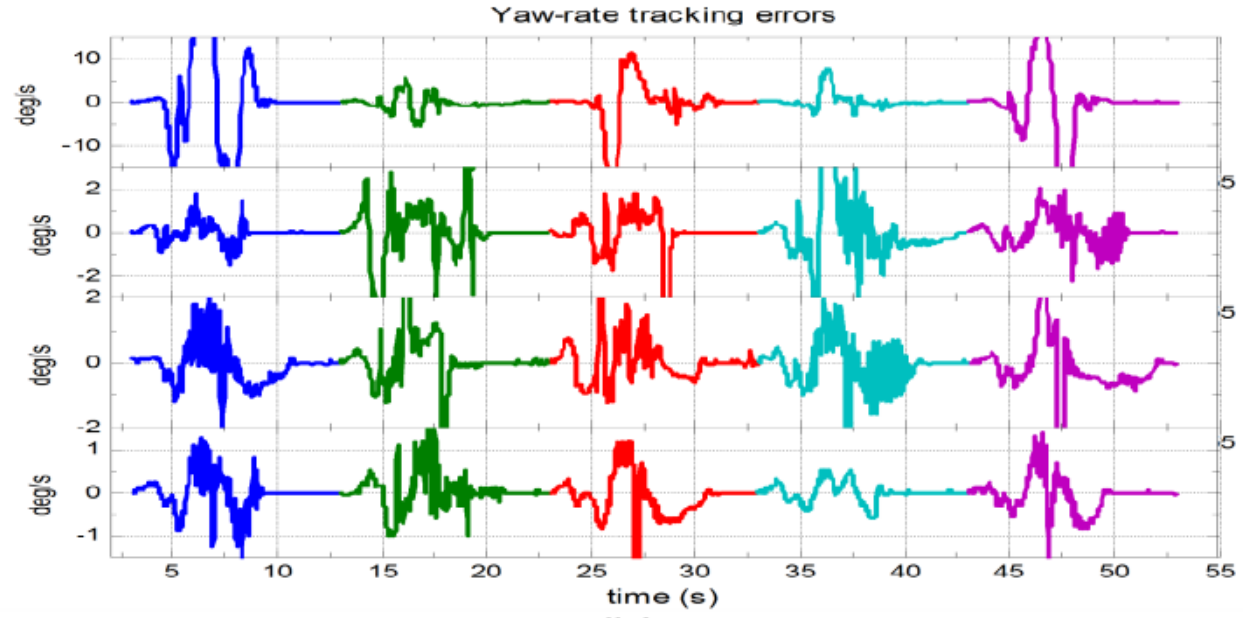

(b)

Figure 7: Yaw-rate responses for the case of the inexperienced driver: (a) yaw rates and target yaw rates, (b) yaw rate tracking errors. 
To further examine driver effect on the lateral dynamic of the SUV, the simulation results of 20. DLC test runs by the experienced driver are analyzed. Figure 8 shows the yaw rates, target yaw rates, and tracking errors of the 20 runs. As expected, a lower peak yaw rate has been observed since the beginning of the 20 test runs. Less variation of the peak yaw rate has been found throughout the 20 test runs, except four runs with intended driver inputs. This indicates that an experienced driver is more capable of handling a vehicle in emergency situations in such a way that a less-demanding target is generated and a better tracking performance can be expected from the DBC controller. The worst peak yaw rate tracking error has been lowered from 15deg/ $\mathrm{s}$ for the inexperienced driver (Figure 7 ) to $5 \mathrm{deg} / \mathrm{s}$ for the experienced driver (Figure 8), except for the four special runs (i.e., last two runs in groups 3 and 4). The target yaw rates of the intended inputs show the same level of amplitudes as others, but exhibit much poorer tracking performance. This fact indicates that the performance of the controlled SUV is not dependent on the amplitude of the target trajectory.
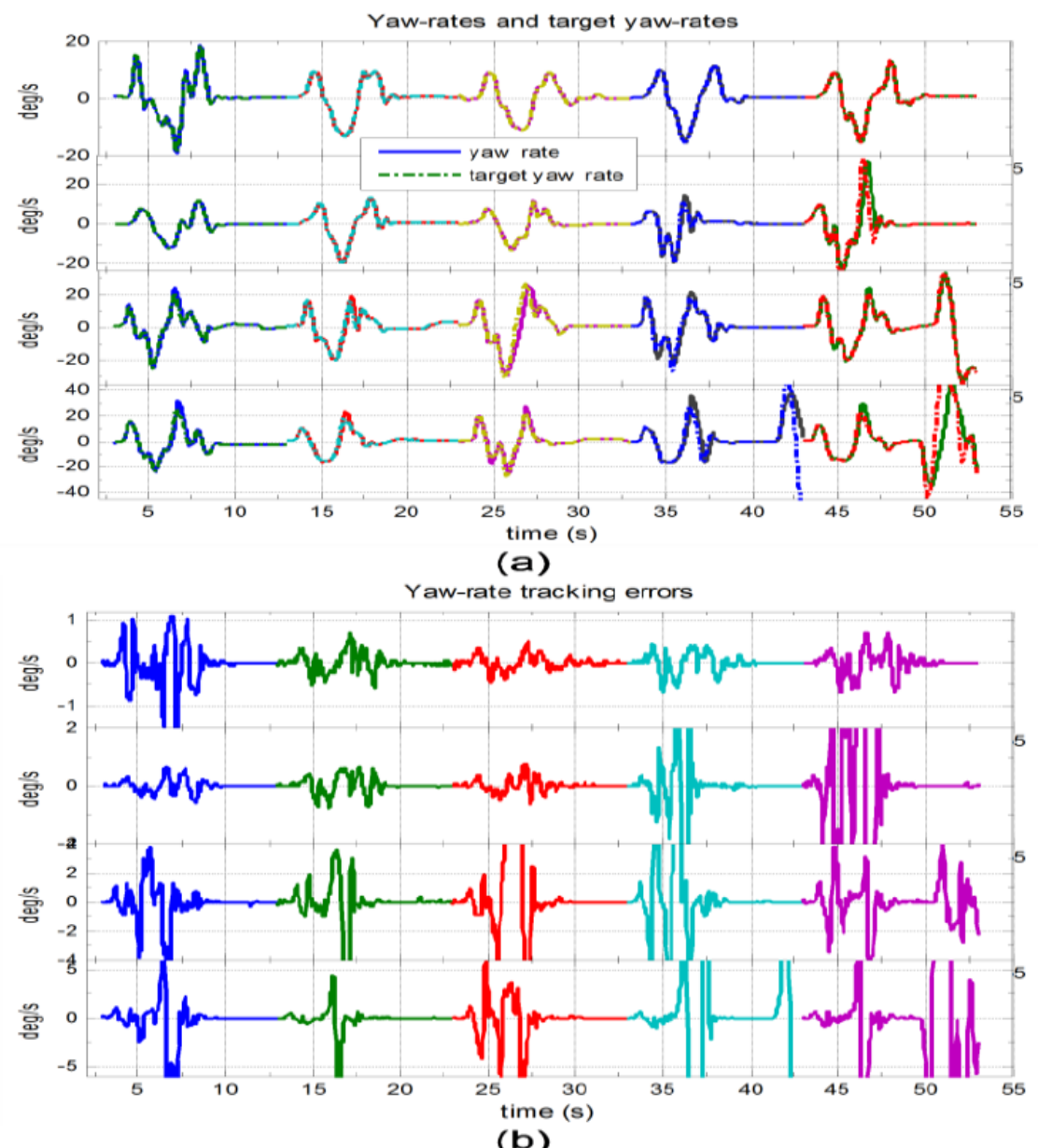

(b)

Figure 8: Yaw-rate responses for the case of the experienced driver: (a) yaw rates and target yaw rates, (b) yaw rate tracking errors.

The yaw rate data of different drivers may be evaluated using frequency analysis. The power spectral densities (PSDs) of target yaw rate trajectories from both the drivers are seen in Figure 9. The high peak tracking errors found in Figures 7 and 8 are directly related with high frequency contents in the target yaw rate trajectories. This observation is consistent with the expectation that an effective controller should exhibit good performance in 
low frequency range without tracking high frequency content. Since high frequency contents do not occur in the target trajectories generated by the experienced driver, except for the four intended operations. Good tracking performance has been achieved in the operations by the experienced driver. The difference between an inexperienced driver and an experienced driver is that an experienced driver can effectively prevent from generating high frequency contents in the targets.

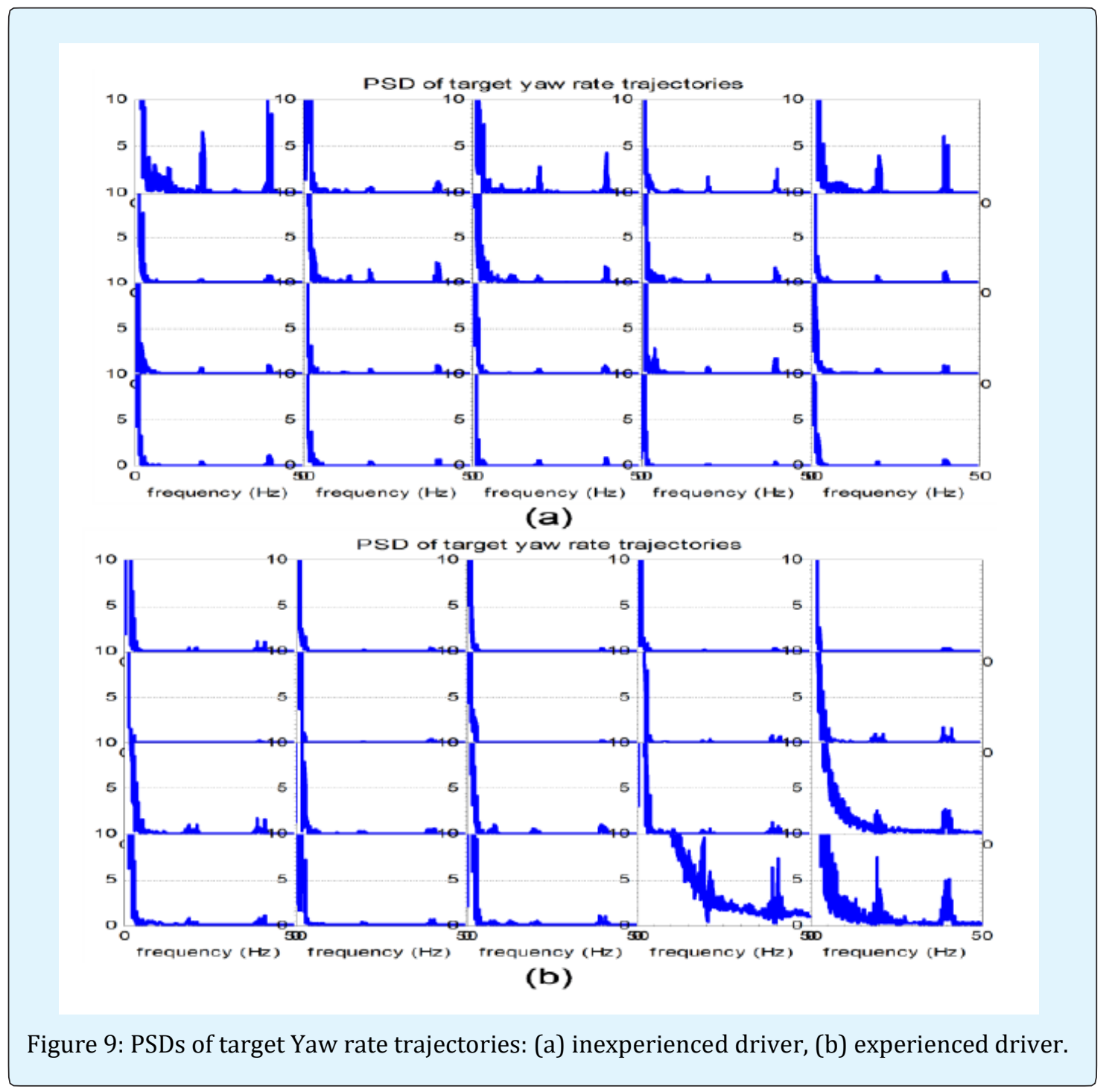

High frequency contents in the target trajectories are detrimental from the view of control. They raise the difficulty of a tracking controller and humiliate the performance of a controlled system. In the current situation, the target trajectory is produced in on-line simulations based on the input from the driver. The experiments have shown that, during an emergency scenario, high frequency contents are more likely generated by an inexperienced driver (Figure 9). Even equipped with an advanced ESC, the driver still plays an important role. To alleviate the dependence of SUV performance on the driver actions, a low-pass filter may be used to remove the high frequency contents. A lowpass filter with $15 \mathrm{~Hz}$ cut-off frequency is used to the yaw rate target trajectory. Shown in Figure 10 are the resulting yaw rates and target yaw rates with the filter. Similar tracking performances have been achieved for both the drivers. With appropriate filters, the performance difference of the controlled SUV driven by different drivers becomes non-evident. 


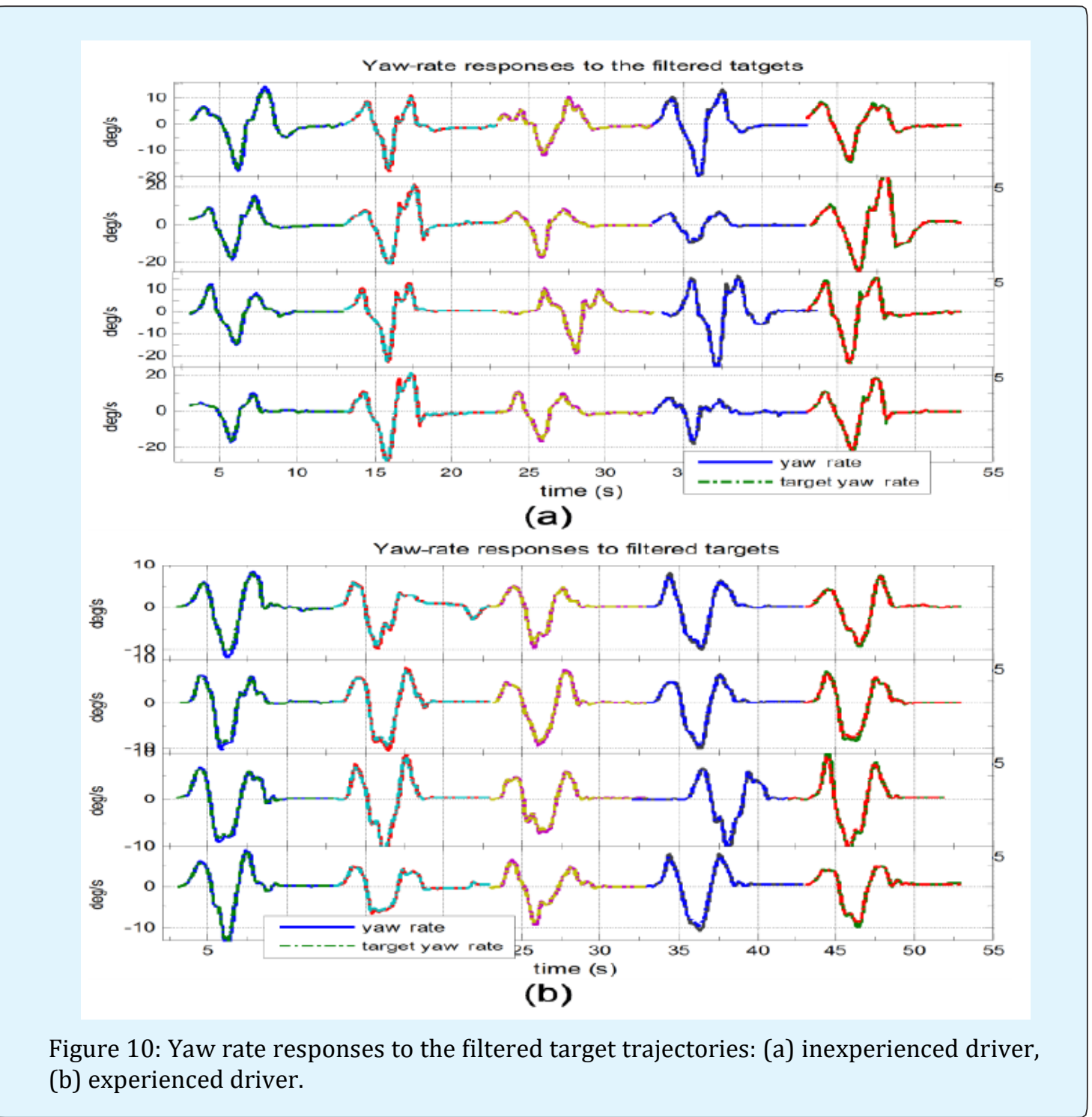

DLC Simulations for controller robustness: In practice, a vehicle frequently faces parametric uncertainties and variable operating conditions. Among various parametric uncertainties, the sprung mass variation is a typical case, which is difficult to model mainly due to payload variation. An important operating parameter for external environment is frictional coefficient $(\mu)$ of road-wheel interface. To examine the robustness of the SUV with DBC to the variation of external environment, a series of experiments with the frictional coefficient taking the value of $0.2,0.5$, and 0.85 , representing a low-, medium-, and high-frictional road surface, respectively, have been conducted by the two drivers under the DLC maneuver. In the nominal case, the frictional coefficient takes the value of 0.85 .
On the low-frictional road, i.e. $\mu=0.2$, the performance of the SUV with DBC is significantly improved over the baseline vehicle in terms of yaw rate. However, much larger sideslip angles exhibit compared against the target sideslip angles. The gap between the performances of the SUV driven by different drivers is not evident. Due to the low friction, the operation of the SUV with DBC is very fragile. An attention must be paid when conducting a test maneuver. Stability loss can be easily triggered by a large yaw rate. The yaw rate of the SUV should be strictly constrained to a range large enough to execute the maneuver. With the jointed effort of the controller and driver, a good tracking of the target yaw rate is achievable with a large sideslip angle error. 
On the medium-frictional road, i.e. $\mu=0.5$, the performance of the SUV with DBC is superior to the baseline design, and exhibits further improvement over the operation on the low-frictional road. An excellent tracking of target yaw rate is achievable. Larger sideslip angles than the targets tend to reduce error with respect to the cases in the low $\mu$ operation. Again, the performance gap between the test runs by different drivers is not evident.
Finally, on the high-frictional road, i.e. $\mu=0.85$, excellent tracking of the yaw rate has been achieved by both of the drivers. Continuous improvement in terms of sideslip angle has been attained by both of the drivers, with amplitudes of sideslip angles reduced below that of the targets. Even though, the performance gap of the test runs by different drivers is not apparent, the performance improvement in terms of sideslip angle of the experienced driver is more evident than that of the inexperienced driver as seen in Figure 11.

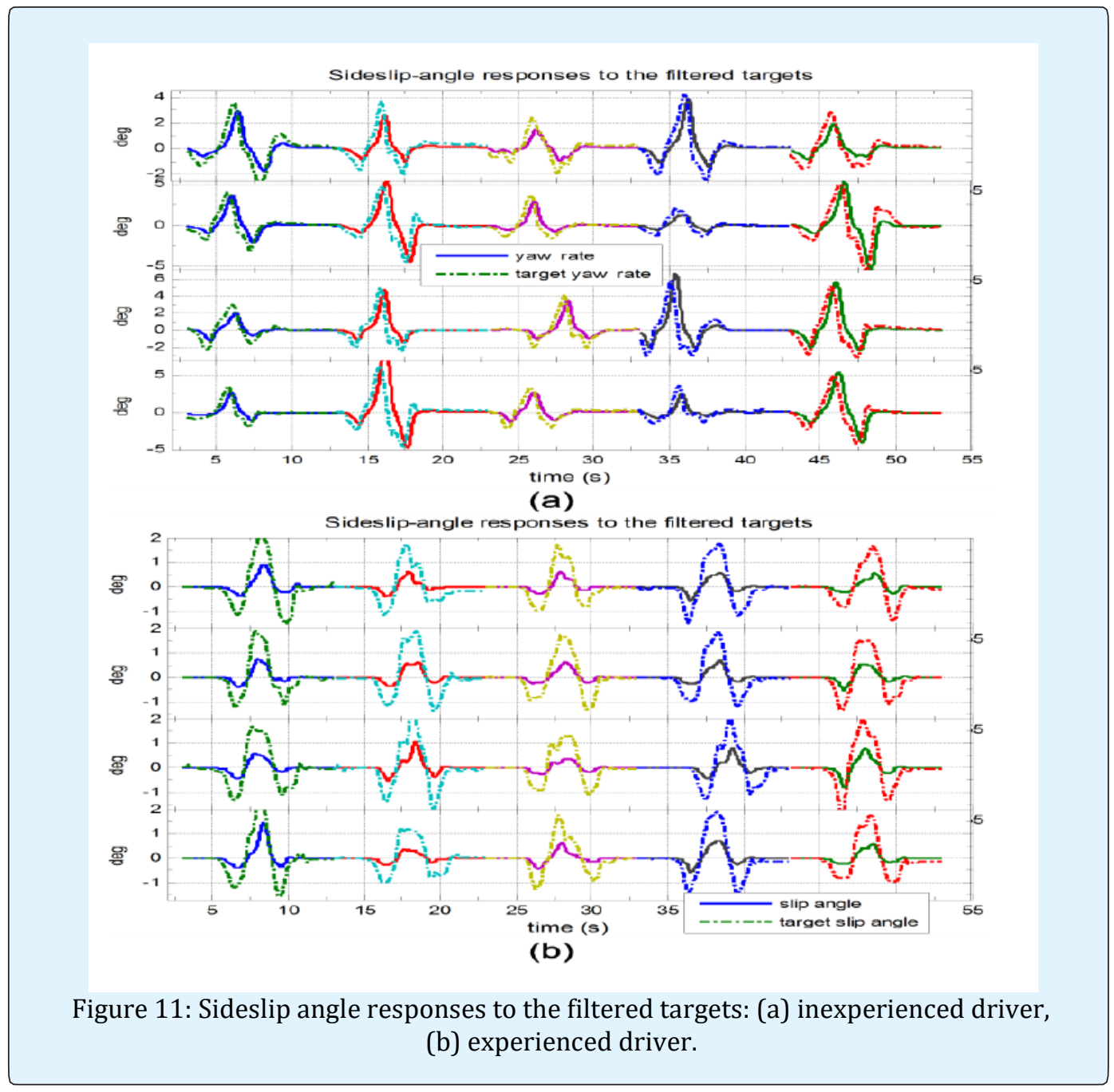

The DSIL real-time simulations indicate that the SMC controller has attained robustness on a wide range of roads with low to high friction coefficients in both the cases of the inexperienced and experienced drivers. Good tracking of yaw rate is achievable on varied road frictional conditions. The sideslip angle, however, varies with the friction coefficient of the road, that is, the sideslip angle of the SUV with DBC decreases with the increment of the road friction coefficient. The performance gap of the test runs by different drivers becomes more evident in terms of the sideslip angle in high friction road operation. A lower slip angle can be expected for an experienced driver. 
Simulated accident avoidance test: The accident avoidance (AA) procedure is specified as follows. Two vehicles, which are traveling at $130 \mathrm{~km} / \mathrm{h}$ on a straight road with a friction coefficient of $\mu=0.85$, is $16.8 \mathrm{~m}$ apart. The leading vehicle stops randomly, and the trailing one executes an immediate DLC maneuver to avoid crash by traversing to the left lane with the friction coefficient of $\mu=0.5$, then returning to the original lane. The trailing vehicle is of interest. The objective of the AA procedure is to examine the dynamic features of the interaction of driver-vehicle-controller-road. The deviation of the trajectory of the left wheels of the leading vehicle from that of the right wheels of the trailing vehicle can be used to verify the success of the test maneuver. In other words, without overlapping of the trajectories and running off the road indicates a successful AA test. The transient response of the vehicle of interest can be used to extract information of the directional performance of the vehicle considering the interaction of driver-vehicle-controllerroad under the specific procedure.
Figure 12 illustrates the relationship of the lateral acceleration (Ay) versus the SWA of the SUV, and the trajectory of front-left wheel of the vehicle derived from ten simulated AA tests by the experienced driver. There is no significant difference in the rising portion of the AySWA curves between the controlled and baseline vehicles. This may be attributed to the fact that the human driver's steering action precedes the controller's action with a large time interval. After an initial SWA peak, the magnitude of Ay per Ay-SWA curve is substantially lower in the case of the controlled vehicle than that of the baseline vehicle. This may be explained by the driver's over-reaction. In a panic situation, as is evident with the test maneuver, the driver may input large steer angle. The simulation results from different drivers show that a driver's over-reaction is largely dependent on the driver's experience. Higher over-reaction is more likely imposed by an inexperienced driver. Simulation results seen in Figure 12 reveal that the DBC controller can effectively improve the lateral stability of the SUV in the scenario of the human driver's over-reaction by suppressing the undesired extreme lateral and yaw accelerations.

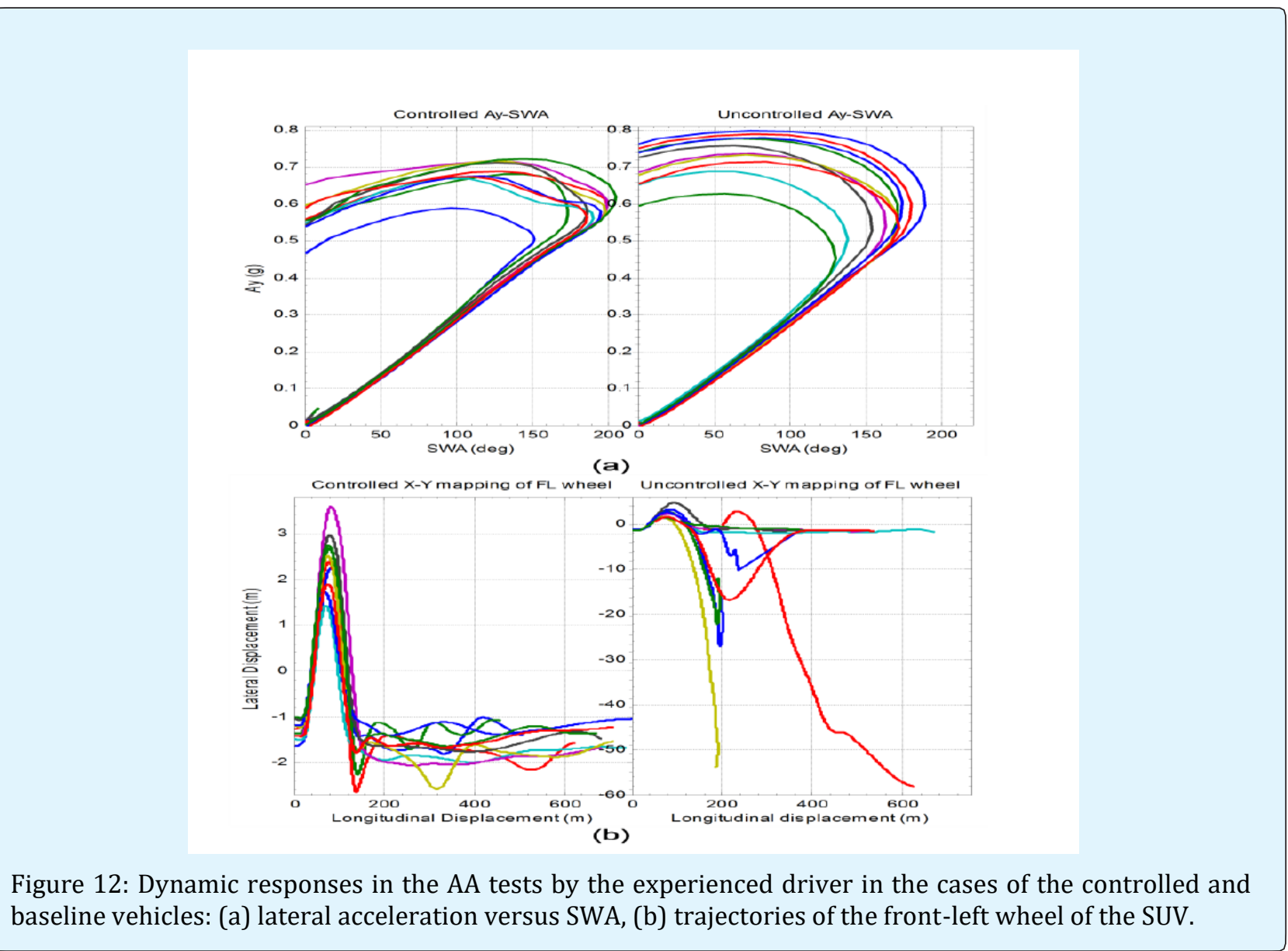

Shenjin Zhu and Yuping He. Design and Validation of Differential Braking Controllers for Sport Utility Vehicles Considering the Interactions of Driver and Control System. 


\section{Ergonomics International Journal}

The trajectories of the front-left wheel of the SUV shown in Figure 12 demonstrate that with the DBC controller, all ten test runs have successfully avoided crashing into the suddenly stopped leading vehicle, and the DLC maneuver is successfully executed. The DSIL realtime simulation results also show that the experienced driver achieves $40 \%$ success rate of the ten AA tests in the case of the baseline vehicle. In contrast, the inexperienced driver experiences $100 \%$ failure rate of the ten AA tests in the case of the baseline vehicle. In other words, with severe over-reaction of the inexperienced driver, the baseline vehicle runs off the road, spins out, and loses lateral stability. In the case of the SUV with DBC, similar real-time simulations involved the inexperienced driver are conducted, and the results show that a $100 \%$ success rate is attained with the aid of the DBC controller. The uniformity of the trajectories due to the DBC controller is apparent for both of the drivers, whereas the trajectories in the case of the baseline vehicle are highly non-uniform. Many of the failed test runs by the experienced driver result in spin-out, and those, which do not spin out, experience an off-road excursion (a major collision with the leading vehicle). The inexperienced driver significantly worsens this situation.

\section{Conclusions}

This paper presents a design and validation of a DBC controller for SUVs using DSIL real-time simulations. A nonlinear yaw-plane model is generated to derive the nonlinear DBC controller, which tracks the target trajectories defined by using the bicycle model. The effectiveness of the controller is assessed using the sinewith-dwell test maneuver specified by FMVSS 126 . To further validate and improve the controller design, the real-time version of the controller and the CarSim-based SUV model are reconstructed; with the integration of the controller and SUV model, the real-time simulations are implemented on the UOIT DSIL platform.

Simulation results illustrate that the DBC controller can effectively manipulate the yaw moment to improve the lateral stability of the SUV. With the effective coordination between the driver and the controller, the overall performance of the SUV can be improved. The driver plays an imperative role in the overall performance of road vehicles. Substantial performance gap of the vehicle driven by different drivers has been identified, and alternative solutions to minimize the gap are proposed. With the DSIL real-time simulations, the interactions among the driver, DBC controller, and SUV model are well exposed and can be fully examined for improving designs. The DSIL platform provides a cost-effective method for vehicle stability control system evaluation prior to invehicle road tests.

\section{References}

1. Zong C, Zhu T, Wang C, Liu H (2012) Multi-Object Stability Control Algorithm of Heavy Tractor Semitrailer based on Differential Braking. Chinese Journal of Mechanical Engineering 25(1): 1-10.

2. Chen B, Peng H (2001) Differential-Braking-Based Rollover Prevention for Sport Utility Vehicles with Human-in-the-Loop Evaluations. Vehicle System Dynamics 36(4-5): 359-389.

3. US Department of Transportation Office of Public Affairs (1999) DOT Requires Upgraded Rollover Warning Label for Sports Utility Vehicles, Press Releases, No. NHTSA 8-99.

4. Sun Z, Chen SK (2010) Automotive Active Safety Systems. IEEE Control Systems Magazine 30(4): 3637.

5. Sun T, He Y (2012) Lateral Stability Improvement of Car-Trailer Systems Using Active Trailer Braking Control. Journal of Mechanics Engineering and Automation 2(9): 555-562.

6. Palkovics L, Fries A (2001) Intelligent Electronic Systems in Commercial Vehicles for Enhanced Traffic Safety. Vehicle System Dynamics 35(4-5): 227-289.

7. Rajamani R (2006) Vehicle Dynamics and Control, Springer, New York.

8. Van-Zanten AT, Gmbh RB (2002) Evolution of Electronic Control Systems for Improving the Vehicle Dynamic Behavior. Proc Int Symp Advanced Vehicle Control 20: 7-15.

9. Zhang SQ, Zhang TX, Zhou SW (2009) Vehicle Stability Control Strategy based on Active Torque Distribution and Differential Braking. Proceedings of International Conference on Measuring Technology and Mechatronics Automation, pp: 922-925.

10. Barbarisi O, Palmieri G, Scala S, Glielmo L (2009) LTVMPC for Yaw Rate Control and Side Slip Control with Dynamically Constrained Differential Braking. European Journal of Control 15(3-4): 468-479. 
11. MacAdam C, Ervin R (1998) Differential Braking for Limited-Authority Lateral Maneuvering. IDEA Program Final Report, Report No. UMTRI-98-43.

12. Friedman K, Hutchinson J (2009) Review of Existing Repeatable Vehicle Rollover Dynamic Physical Testing Methods. Proceedings of ASME International Mechanical Engineering Congress and Exposition 16: 51-59.

13. Short M, Pont MJ (2008) Assessment of High-Integrity Embedded Automotive Control Systems Using Hardware in the Loop Simulation. Journal of Systems \& Software 81(7): 1163-1183.
14. Rajamani R (2012) Vehicle Dynamics and Control $2^{\text {nd }}$ (Edn.), Springer.

15. VS Solver Programs Reference Manual (2011) CarSim 8.1 Help Manual.

16. Slotine J, Li W (1991) Applied Nonlinear Control. Prentice- Hall Inc.

17. US Department of Transportation National Highway Traffic Safety Administration Laboratory Test. Procedure for FMVSS No. 126, Electronic Stability Control Systems.

18. Sine with Dwell Test in CarSim. 